\title{
Structural Changes of Adhesive Discs during Attachment of Boston Ivy
}

\author{
*Correspondence to: \\ Kim IS, \\ Tel: +82-53-580-5305 \\ Fax: +82-53-580-5305 \\ E-mail: botany@kmu.ac.kr \\ Received September 25, 2014 \\ Revised November 25, 2014 \\ Accepted November 26, 2014
}

\begin{abstract}
This study investigates the developmental pattern of adhesive discs (ADs) to highlight the ontogeny and structural changes that occur during the growth of Boston ivy. Initiation to postmortem features of ADs were examined through light and scanning electron microscopy. The study also reveals a new finding of the dislocation of peripheral tissues of adaxial origin. Four phases of attachment are suggested with regards to its climbing behavior: 1) pre-attachment, 2) upon attachment, 3) after attachment, and 4) final attachment. During initiation, several ADs originate from tendril primordia without epidermal differentiation. However, different growth rates in the epidermis results in completely different ADs. ADs were discerned by size, shape, and color during expansion, but cells in the adaxial surface remained alive longer than the other side. Upon contact, the ADs demonstrate simultaneous growth and deterioration, but once attachment is established the latter process subdues to final stages. Epidermal transformation, adhesive secretion, cellular disruption, and mechanical stress were essential for the self-clinging nature of Boston ivy. The post-attachment sequence is also believed to be critical in achieving maximum mechanical strength to provide extensive support. The developmental process of $\mathrm{ADs}$ is prompted by tactile stimulation but in a highly organized and systematic manner.
\end{abstract}

Key Words: Adhesive disc, Attachment process, Boston ivy, Epidermis, Structural changes

\section{INTRODUCTION}

Boston ivy, Parthenocissus tricuspidata, is a commonly cultivated, ornamental vine that is characterized by its climbing behavior with tendrils along its shoots. A tendril primordium initiates from the shoot apical meristem peripherally and develops through lateral growth (Wilson \& Posluszny, 2003). The tendrils exhibit thigmotropic properties that help the plant adhere onto substrates laterally and/or vertically. During active growth, tendrils most commonly develop adhesive discs (ADs) at their tips while tips that do not produce ADs often disintegrate (Gerrath \& Posluszny, 1989).

Tactile stimulation from substrates in the vicinity of tendrils has been considered a crucial factor in the growth of the plant (Endress \& Thomson, 1977; Jiang, 1994; Yim \& Kim, 2002;
Wilson \& Posluszny, 2003; Deng, 2008a, 2008b; Lee \& Kim, 2011). In fact, this stimulus is fundamental in initiating a series of structural changes within ADs. Early in development, bilaterally-arranged and relatively symmetric ADs are formed with stimulation. Adhesive materials secreted from ADs for attachment are also speculated to play an important role in these changes (Junker, 1976; Endress \& Thomson, 1977; Ragni et al., 1988; Jiang, 1994; Yim \& Kim, 2002; Kim \& Kim, 2007; Bowling \& Vaughn, 2008; Deng, 2008a, 2008b; He et al., 2010a, 2010b; Lee \& Kim, 2011).

The two phases of pre- and final attachment have been proposed to be responsible for the adhering process of Parthenocissus (Steinbrecher et al., 2009). In a study of ontogenetic variations in morphology and attachment of Parthenocissus ADs, Steinbrecher et al. (2009) distinguished the pre-attachment phase from the final attachment phase

(a) This is an open-access article distributed under the terms of the Creative Commons Attribution Non-Commercial License (http://creativecommons.org/licenses/by-nc/3.0) which permits unrestricted noncommercial use, distribution, and reproduction in any medium, provided the original work is properly cited.

Copyrights @ 2014 by Korean Society of Microscopy 
through different features of development, initial vs. final contact, tissue lignification, and $\mathrm{AD}$ attachment strength. Functional development of ADs begins with positive thigmotropism during initiation, but their supporting role is retained beyond termination. Therefore, when exploring structural aspects of Boston ivy the pre-attachment phase should include primordial stages of ADs, and the final attachment phase should include postmortem stages. Because tissue lignification and mechanical stress of $\mathrm{ADs}$ sustain both growing branches and dead ones from the previous year(s), their long-term effects should be taken into consideration for structural analysis.

Preliminary survey on the growth pattern of ADs have revealed that their structural differentiation from initiation to completion is rapid but systematic. Thus, the present study includes from the initiation to postmortem stages to investigate this phenomenon using both light and electron microscopy. In addition to shining light on the ontogeny and resulting development of ADs, it provides new information regarding dislocated peripheral tissue of abaxial origin.

\section{MATERIALS AND METHODS}

Specimens of Boston ivy grown on brick walls of several buildings were collected from March to November in the years of 2012 and 2013. Sample sites were located in the Sungseo campus of Keimyung University in Daegu, Korea. Approximately 30 to 50 tissue samples of shoot tips, tendrils, and various stages of ADs were used for ontogeny and structural analyses for this study.

Tissue samples to study morphological and anatomical aspects of ADs were processed using microscopy procedures for Parthenocissus (Lee \& Kim, 2011). The samples were fixed for two to four hours in 3\% glutaraldehyde and rinsed with $0.1 \mathrm{M}$ sodium phosphate buffer ( $\mathrm{pH}$ 6.8 7.2). After rinsing the tissues three times with the same buffer, they were postfixed overnight using osmium tetroxide. Dehydration using a graded series of acetone was followed by gradual infiltration with a low-viscosity resin mixture and insertion into pure resins. Sections about 0.8 to $1.5 \mu \mathrm{m}$ in thickness were made and stained with $0.3 \%$ toluidine blue. Then, they were examined with Zeiss Photomicroscope II (Zeiss, Germany) in order to compare the $\mathrm{AD}$ tissue differentiation during development.

For scanning electron microscopy, tissue samples were processed following procedures of Kim \& Kim (2007). The tissues were fixed for three hours in 3\% glutaraldehyde at room temperature and washed three times in $0.1 \mathrm{M}$ sodium phosphate buffer ( $\mathrm{pH} 7.2$ ). After washing with the buffer, they were fixed in 2\% 4\% osmium tetroxide for two to 12 hours at $4^{\circ} \mathrm{C}$. These post-fixed samples were dehydrated in a graded series of acetone, then dried with liquid carbon dioxide by Emitech K850 CPD (Emitech Ltd., UK). Processed samples were mounted directly onto stubs using a doublesided adhesive tape and were coated with gold/palladium in a sputter coater (Emitech K550X; Emitech Ltd.). Finally, coated samples were examined with Hitachi S-4200 scanning electron microscope (SEM) at Daegu Center, Korea Basic Science Institute in Daegu, Korea.

\section{RESULTS AND DISCUSSION}

Structural differentiation of Boston ivy ADs was prompted by positive thigmotropism of tendrils during development. Several AD primordia originated from a tendril primordium located opposite to the leaf. Once they were exposed to the outside environment and able to detect tactile stimulus nearby, the ADs differentiated rapidly and successful attachment onto the substrate took place within a short period of time. Formation of tendril primordia continued along the shoot. This led the branches to extend considerably to provide a strong support. Four phases of attachment were apparent in its climbing behavior: 1) pre-attachment, 2) upon attachment, 3) after attachment, and 4) final attachment.

\section{Pre-attachment and upon Contact}

During initiation, the tendril tip became swollen and formed a globular tendril primordium from which approximately five to seven $\mathrm{ADs}$ originated, each covered by bracts. $\mathrm{ADs}$ within the bracts were usually 100 to $300 \mu \mathrm{m}$, undifferentiated, and oval to circular in shape (Fig. 1A). Immature ADs were light green in color, the adaxial and abaxial epidermis were still indistinguishable. The stomata were paracytic and approximately $11 \sim 13 \times 15 \sim 18 \mu \mathrm{m}$ (width $\times$ length) in size (Fig. 1B). ADs formed in clusters at the onset, but with thigmotropism they soon arranged themselves in a bilateral pattern. Young ADs developed into adaxially inflated semispherical disc structures approximately 600 to $800 \mu \mathrm{m}$ in active state (Fig. 1C). Tendril contact with a substrate was required for disc development. Branches with $\mathrm{ADs}$ that were unsuccessful in making contact gradually dried up and died. However, an attachment failure was hardly found in this study if a substrate was simply in the vicinity of ADs.

\section{Upon Attachment and after Attachment}

Varying growth rates in epidermal layers resulted in an adaxial convex and abaxial concave configuration (Fig. 1D) among actively growing $\mathrm{ADs}$ with a green hue. Prior to stimulation, a secreted material, presumably adhesive in nature, accumulated in the concaved area. This material was soon released to cover the entire central zone. Yet epidermal cells in the peripheral zone undertook sizeable changes to form numerous papillae (Fig. 1E). Rapid cell divisions in the peripheral epidermis yielded papillae that transformed into numerous finger-like 
projections before and/or upon contact. These projections were pushed toward the contact surface, possibly by the discharged adhesive that caused the central zone to flatten (Fig. 1F). The hardened adhesive spread extensively to penetrate deep into the projections, enabling ADs to adhere for support. Between $\mathrm{ADs}$ and the substrate, this adhesive filled not only the interfacial region but also neighboring projections (Fig. 1G). Overflowing secretion creating a heavily coated surface was frequently noted in the abaxial side. It has been well accepted that the adhesive contained certain components that function as cementing and hardening agent.
Considering secretion during attachment of Boston ivy, mucilage, polysaccharide, or tannin have been suggested as the adhesive constituent (Endress \& Thomson, 1976; Yim \& Kim, 2002; Deng, 2008a; Steinbrecher et al., 2009). Chemical composition with organic components including metal elements has also been reported by He et al. (2010b). Given current data, structural differentiation in abaxial ADs ensue in a temporally and spatially different mode, although it appears to be a seemingly synchronized process.

A corresponding growth took place simultaneously in the adaxial surface upon stimulus. Rapid cell divisions in the
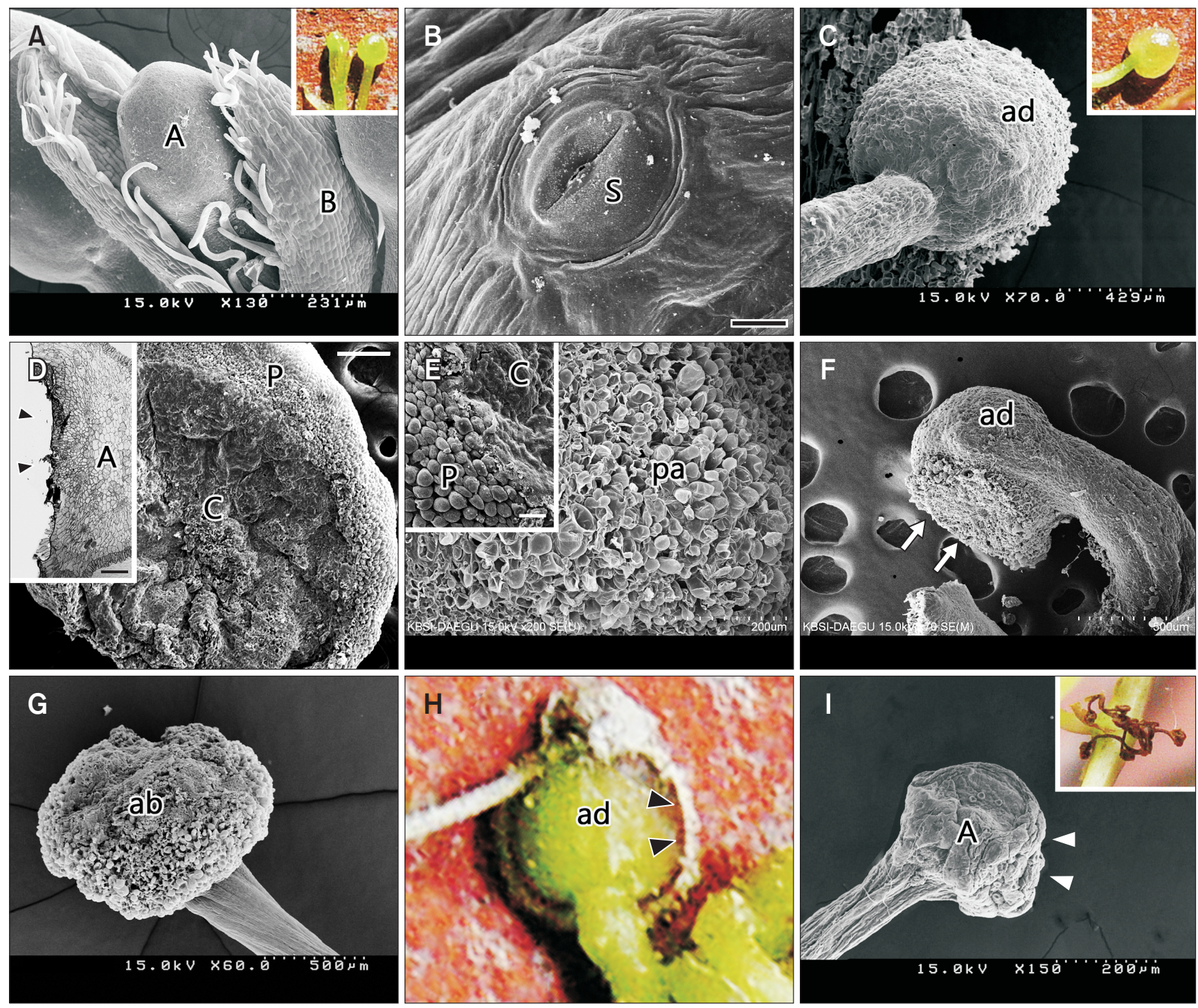

Fig. 1. Adhesive discs (ADs) in the pre-attachment phase and upon contact. (A) Several ADs within bracts at initiation. A, AD; B, bract. Inset: Three ADs in cluster. (B) Relatively small stoma (S) in AD. Scale bar=6.0 $\mu \mathrm{m}$. (C) Adaxially inflated young AD. ad, adaxial surface. Inset: Disc-shaped young AD upon contact. (D) Concave abaxial surface. C, central zone; P, peripheral zone. Scale bar=80 $\mu \mathrm{m}$. Inset: Cross section of developing AD. Arrowheads indicated concave central zone. Scale bar=150 $\mu \mathrm{m}$. (E). Part of the peripheral zone with innumerous papillae (pa) prior to stimulation. Inset: Close up view of central zone (C) with peripheral zone (P). Scale bar=25 $\mu \mathrm{m}$. (F) Flattened central zone (arrows) with intact ad. (G) Secretion of adhesive materials completely filled the abaxial surface $(\mathrm{ab})$. (H) Two zones clearly distinguished by the color (arrowheads). (I) Deteriorating AD (arrowheads). Inset: Several ADs deformed. 
adaxial epidermis made ADs fully enlarged and matured. In particular, careful examination of the upper surface of ADs revealed two zones clearly by their color: central green with red margins to brown periphery with whitish grey margins (Fig. $1 \mathrm{H}$ ). However, only ADs that were able to detect mechanical or tactile stimulation underwent sequential differentiation. Upon contact, ADs as small as $500 \mu \mathrm{m}$ proceeded to a series of morphological changes as mentioned previously. In the absence of thigmotrophic stimulation, structural modification did not occur even in $\mathrm{ADs}$ larger than $800 \mu \mathrm{m}$. Tissues of unattached ADs began to shrink, become brown, and deteriorate (Fig. 1I). Abscission of unattached ADs (Wilson \& Posluszny, 2003), however, was not detected in this study.

In the inflated adaxial surface, two zones began to demarcate (Fig. 2A) as in the abaxial surface; a central zone encircled by a peripheral zone (Fig. 2B). The central and peripheral zones likely coincided with two zones illustrated in abaxial surface of Fig. 1D and F. Surprisingly, the periphery consisted of numerous finger-like projections that originated from the abaxial peripheral zone, although flattened and considerably shrunken compared to those in the abaxial surface. The ADs
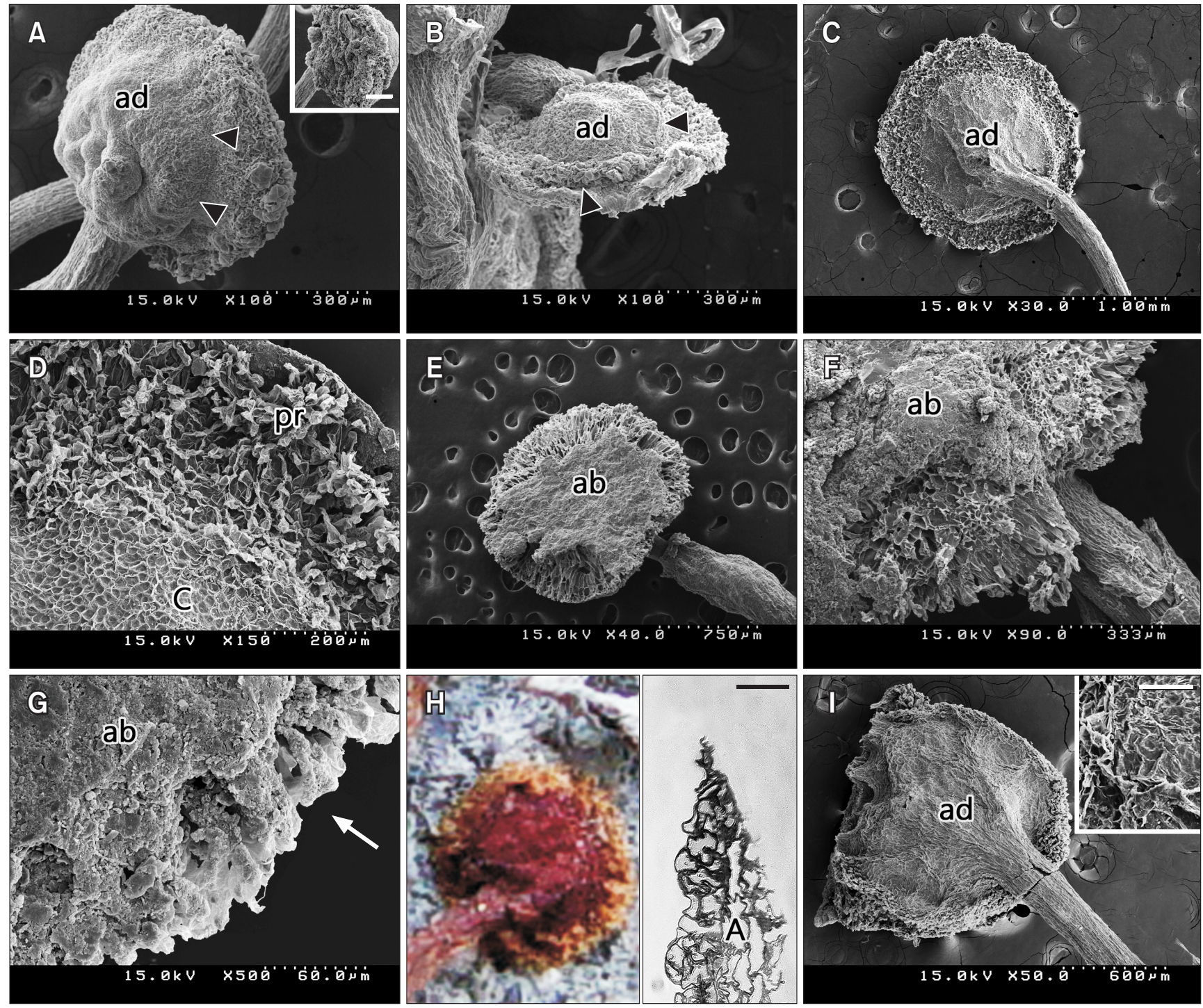

Fig. 2. Adhesive discs (ADs) in the after and final attachment phase. (A) Early demarcation of the two zones (arrowheads) in the adaxial surface (ad). Inset: AD. Scale bar $=200 \mu \mathrm{m}$. (B) Expansion of the peripheral zone (arrowheads). (C) Fully matured AD. Note the dried ad. (D) Part of the central zone (C) encircled by peripheral zone with numerous projections (pr). (E) Old lignified AD with substrate at the abaxial surface (ab). (F, G) Part of exceptionally hardened intertwined abaxial surface. Adhesive thickly covered the entire surface. AD cells were not visible even at the periphery (arrow). (H) Withered AD in the postmortem phase. Inset: Cross section of dead adhesive disc (A). Scale bar $=400 \mu \mathrm{m}$. (I) AD from dead branch. Inset: Close up view of ad in Fig. I. Scale bar $=100 \mu \mathrm{m}$. 
at this phase yielded a high degree of mechanical support and were approximately $1.5 \sim 2.5 \mathrm{~mm}$ in size (Fig. $2 \mathrm{C}$ ). When the abaxial cells released adhesive materials, the interfacial region became intertwined as if it were one unit (Fig. 2D). The chemical reaction of the adhesive spread extensively in the interfacial and intercellular regions, playing an important role in providing stronger absorbability (He et al., 2010a, 2010b).

\section{Final Attachment}

Shrinking of the inflated adaxial surface appeared to push the ADs up against the contact surface with the application of constant mechanical stress (Fig. 2C and D). The shrunken $\mathrm{ADs}$ began to lose their vibrant color and became dark brown, showing tissue lignification with apparent signs of aging and death. Such lignified tissues seemed to enable ADs to adhere to a supporting structures even when faced with the death of a branch. Steinbrecher et al. (2009) have found that the attachment strength of young ADs is actually only about $28 \%$ of older lignified ADs (Fig. 2E), indicating the intensification of mechanical forces after attachment.

The pre-attachment phase is followed by structural differentiation with adhesive secretion while the hardening of the adhesive, mechanical stress, tissue lignification, and cell disruption appear to be important elements during and/ or after the final attachment phase. After this phase, attempts to separate $\mathrm{ADs}$ from the surface they are attached to are extremely difficult without causing tissue damage. If separated by pulling the two apart, the $\mathrm{AD}$ surface exhibits fragments of the substrate with only a fraction of living cells. After the final attachment phase, the ADs were nearly all dead with no living cells but had an exceptionally hardened intertwined surface (Fig. 2F and G). Furthermore, early death of ADs play a significant role in lessening the weight of branches in order to maintain robust attachment to a substrate (Fig. $2 \mathrm{H})$. Therefore, the final attachment phase including aging and death, almost certainly triggered the resistance of $\mathrm{AD}$ displacement from substrates by external forces (Endress \& Thomson, 1976; Deng, 2008a; He et al., 2010a). In plants that lack self-clinging behavior, aged and dead tissues are usually separated and removed by abscission from the plant. However, the ADs of Boston ivy can remain on the substrate vertically and laterally for a long period, up to several years. This implies that they serve a postmortem function of lightening the weight of dead branches (Fig. $2 \mathrm{H}$ and I).

Therefore, mechanical or tactile stimulation, not the size of ADs, leads immature ADs to continue successive differentiation at a cellular level. The present study is in agreement with previous reports that the stimulus proves to be the most crucial factor for successful attachment of Boston ivy (Endress \& Thomson, 1976, 1977; Junker, 1976; Jiang, 1994; Yim \& Kim, 2002; Kim \& Kim, 2007; Deng, 2008a, 2008b; He et al., 2010a, 2010b; Lee \& Kim, 2011). However, previous studies have not dealt with the occurrence or origin of adaxial peripheral projections in developing Boston ivy ADs. Research by Junker (1976) and Deng (2008a, 2008b) include SEM micrographs of papillae and briefly discuss the growth pattern of the abaxial surface but do not mention further examination regarding subsequent transformation or dislocation. Occurrence of abaxially originated finger-like projections in the adaxial surface indicates that mechanical pressure is substantial when ADs are pushed up against the substrate during attachment. Deng (2008a) has revealed that a single mature $\mathrm{AD}$ can exhibit a weight of $0.5 \mathrm{mg}$, an attached area of $1.22 \mathrm{~mm}^{2}$, an adhesive force of $13.7 \mathrm{~N}$, and a supporting capability that is 260 times greater than its own weight. A maximum pulling force that is two million times greater than its own weight has been measured from a mature $\mathrm{AD}$ as well (Deng, 2008a).

If such a pulling force is necessary to separate a fully grown $\mathrm{AD}$ from the substrate, the $\mathrm{AD}$ likely creates a strong adhesive binding and adds substantial mechanical force to its contact surface while young. Deng (2008a) has hypothesized that the strong adhesive property, microstructures, and mechanical forces of an $\mathrm{AD}$ play a major role in its adhesion mechanism at a cellular and molecular level. This hypothesis is highly possible considering the structural and developmental patterns of an $\mathrm{AD}$.

The hardened adhesive as well as mechanical stress should be treated equally as vital elements that contribute to successful attachment of Boston ivy. In addition, the projections during pre-attachment can be cautiously compared to numerous gecko foot hairs, which play an essential role in van der Waals interactions for adherence (Autumn et al., 2002; Gao et al., 2005; Hansen \& Autumn, 2005). Contrary to flexible gecko hairs, however, interactions among the projections in Boston ivy are temporary and stationary. The interactions are likely activated shortly before adhesive hardening and disappear once a successful attachment is established.

\section{CONCLUSIONS}

Structural changes of ADs from initiation to postmortem in Boston ivy plants reveal a well-organized and systematic pattern of development. This study suggests four phases for the adhering process shown in climbing behavior: 1) preattachment, 2) upon attachment, 3) after attachment, and 4) final attachment. Dislocated peripheral tissues of abaxial origin are also a new and intriguing discovery.

Several ADs are derived from tendril primordia without epidermal differentiation at the onset. Varying epidermal growth rates during the pre-attachment phase results in different surfaces displaying adaxial and abaxial configurations. With thigmotropism, secretion of adhesive materials occur in the abaxial central zone, while peripheral 
cells form numerous projections. Subsequent dislocation of peripheral cells come with drastic changes in the intraand extra-cellular level. Cells in the adaxial surface usually remain alive longer than cells on the other side. ADs differ in size, shape, and color during development. Upon contact, ADs undergo both growth and deterioration process at the same time. After attachment, cellular deterioration and death proceed radically, but aged and dead ADs also play important roles in adding strength to the attachment. A series of post-attachment sequence is also believed to be a critical in achieving maximum mechanical strength.

\section{CONFLICT OF INTEREST}

No potential conflict of interest relevant to this article was reported.

\section{REFERENCES}

Autumn K, Sitti M, Liang Y A, Peattie A M, Hansen W R, Sponberg S, Kenny T W, Fearing R, Israelachvili J N, and Full R J (2002) Evidence for van der Waals adhesion in gecko setae. Pro. Natl. Acad. Sci. USA 99, 12252-12256.

Bowling A J and Vaughn K C (2008) Structural and immunocytochemical characterization of the adhesive tendril of Virginia creeper (Parthenocissus quinquefolia [L.] Planch.). Protoplasma 232, 153163.

Deng W (2008a) Parthenocissus inserta: environment-friendly plant or material?: tendril, adhesive disc and adhesive effect. Nat. Sci. Develop. 18, 1220-1225.

Deng W (2008b) Tendril, adhesive disc and super adhesive effect of climbing plant. Nat. Proc. 1, 15.

Endress A G and Thomson W W (1976) Ultrastructural and cytochemical syudies on the developing adhesive disc of Boston ivy tendril. Protoplasma 88, 315-321.

Endress A G and Thomson W W (1977) Adhesion of the Boston ivy tendril. Can. J. Bot. 55, 918-924.

Gao H, Wang X, Yao H, Gorb S N, and Arzt E (2005) Mechanics of hierarchical adhesion structures of geckos. Mech. Mater. 37, 275285.

Gerrath J M and Posluszny U (1989) Morphological development in the Vitaceae. III. Vegetative development in Parthenocissus inserta. Can. J. Bot. 67, 803-816.

Hansen W R and Autumn A (2005) Evidence for self-cleaning in gecko setae. PNAS 102, 385-389.

He T, Miao X, Zhang L, Mu, Y, and Deng W (2010a) Research on the adhesion mechanism of the adhesive disc of Parthenocissus tricuspidata. 18th Int. Vacuum Cong. [Internet]. Available from: http://cpfd.cnki.com.cn/Article/CPFDTOTAL-ZGZU201008001902. htm.

He T, Zhang L, Xin H, and Deng W (2010b) Morphology and mechanics of the adhesive disc of liana Parthenocissus tricuspidata. Pure Appl. Chem. 82, 91-96.

Jiang Z (1994) A morphological study on the wall adhesion mechanism of the adhesive discs of Parthenocissus tricuspidata and P. henriyana. J. Nanjing Agric. Univ. 17, 27-31.

Junker S (1976) A scanning electron microscopic study on the development of tendrils of Parthenocissus tricuspidata and Sieb. \& Zucc. New Phytol. 177, 741-746.

Kim J and Kim I (2007) Epidermal changes of the adhesive disks during wall attachment in Parthenocissus tricuspidata. Korean J. Electron Microsc. 37, 83-91.

Lee M and Kim I (2011) Characteristics of adhesive disks in Parthenocissus tricuspidata during attachment. Korean J. Electron Microsc. 41, 139-145.

Ragni G, Conti G F, Cinti S, and Sapelli PL (1988) A plant model of biological adhesion. Bull. Group Int. Rech. Sci. Stomatol. Odontol. 31, 189-205.

Steinbrecher T, Kraft O, Speck T, Melzer B, and Schwaiger R (2009) Ontogenetic variations in morphology and attachment strength of permanent attachment pads of species of Parthenocissus. 6th Int. Vacuum Plant Biomech. Conf. 446-449.

Wilson T and Posluszny U (2003) Complex tendril branching in two species of Parthenocissus: implications for the vitaceous shoot architecture. Can. J. Bot. 81, 587-597.

Yim J and Kim I (2002) Morphological and cellular characteristics of aerial roots in the epiphytic American ivy (Parthenocissus sp.). Korean J. Electron Microsc. 37, 329-337. 